The MAKING OF A SELF-NEGLECT SEVERITY SCALE

Dyer CB, Pavlik VN, Kelly PA, Lee J, Doody RS, Regev C, Pickens S, Burnett J, Smith SM

Funded in part by the Texas Department of Family Protective Services and the NIH Grant \# P20RR20626

Keywords: self-neglect, environment, assessment 
Acknowledgements: The CREST research team would like to thank all the members of the Texas Elder Abuse and Mistreatment Institute especially James Booker, Carrie Hill, Angela Goins, George Quintero, Vicky Egland, Delphine Baldon, Adriana Cosio-Franco, and Allison Cliatt . We also are grateful for the advice and input of Lori Stiegel, Marilyn Whalen, Holly RamseyKlawsnik, Carolyn Bivens and Paula Mixon. 
The MAKING OF A SELF-NEGLECT SEVERITY SCALE

Dyer CB, Pavlik VN, Kelly PA, Lee J, Doody RS, Regev C, Pickens S, Burnett J, Smith, SM

Abstract

Background: Research in elder self-neglect has lagged behind that of other forms of mistreatment, despite the fact that self-neglect is the most common allegation reported to Adult Protective Service agencies throughout the US. The lack of a gold-standard to measure selfneglect has hampered efforts to study this phenomenon.

Methods: Researchers designed the Self-neglect Severity Scale (SSS) based on interviews with Adult Protective Service workers and a national expert panel. The SSS is based on observation and interview and is administered in the home to include an environmental assessment. It was piloted, extensively field tested and then revised.

Results: The CREST SSS was developed using survey data and consultation with experts in the field. This instrument utilizes observer ratings, interview responses, and assesses subjects’ physical and environmental domains. It also assesses functional status as it relates to health and safety issues. After field and pilot testing the SSS was finalized and is currently undergoing reliability and validity testing.

Conclusions: The CREST SSS was developed as a state scale to provide a common language for describing cases of self-neglect. It is the first self-neglect severity scale available to researchers. If found to be both reliable and valid it can be used in future intervention studies. 


\section{The MAKING OF A SELF-NEGLECT SEVERITY SCALE}

Self-neglect is the most commonly reported allegation to adult protective service agencies in the US.(Pavlik, Festa, Hyman, \& Dyer, 2001; Teaster, Dyer, Mendiondo, Abner, \& Cecil, 2005). Older persons who neglect themselves display abnormalities in a number of domains. They may fail to take medications or seek medical care, even for obvious problems like large tumors or life-threatening conditions. They are impaired in their ability to perform instrumental and basic activities of daily living such as bathing, dressing, cleaning their clothes or their homes or paying their bills. Many have multiple pets, but are unable to care for them. Consequently many of these elders who neglect themselves have poor hygiene, live without utilities and some live in complete squalor. Depression and dementia are also commonly associated risk factors; self-neglect is an independent risk factor for death (Lachs, Williams, O’Brien, Pillemer, \& Charlson, 1998; Dyer, Pavlik, Murphy, \& Hyman, 2000). In 2005 the National Association of Protective Service Administrators investigated over 170,000 cases of self-neglect from 34 states which represented a 34\% increase from 2000. These numbers are only expected to get worse when baby boomers turn 65 beginning in 2011 .

Despite the public health implications of self-neglect, few studies have been published on this topic. There are varying definitions and no gold standard exists for determining the presence or absence of self-neglect (Dyer, Connolly, \& McFeeley, 2002; Fulmer, Dyer, Connolly, \& Guadango, 2004). Although, there are several tools that measure neglect by others, those tools do not fit the syndrome of self-neglect since many rely on caregiver, surrogate or victim histories. Those who neglect themselves usually have no caregivers or surrogates to provide information and they themselves are often cognitively impaired, rendering instruments that rely on self-report inadequate. Also, the tools that measure neglect by others are administered in 
settings such as the hospital or emergency room, and, therefore, fail to include information about the individual's living conditions. These tools are limited to assessment of serious physical appearance at the time of evaluation.

Older persons neglect themselves in ways that may not be discernible on physical examination or outside of the home environment. At present, it is difficult to identify cases of self-neglect other than those reported to social service agencies. Thus, research on self-neglect , addressing its prevalence, risk factors, and on the development and testing of effective interventions for self-neglect, cannot progress until there is a reliable and valid method to detect cases in the general population, and to rate their severity.

We report on the development and pilot testing of a scale to measure the phenomenon of self-neglect. The effort began in the late 1990's as part of collaboration between a research team at Baylor College of Medicine and representatives of the local and state offices of the Adult Protective Services Agency (APS). Subsequently, with funding from the Roadmap Initiative of the NIH, Baylor researchers and their collaborators formed the Consortium for Research in Elder Self-Neglect of Texas (CREST). This funding mechanism supported the pilot-testing, refinement and validation of the Self-neglect Severity Scale (SSS). We report here on the development of the tool, pilot testing and refinement.

\section{METHODS}

The first step in developing the SSS was to identify the domains that should be included in the instrument. The de facto experts in identifying self-neglect are APS specialists who investigate reports and make a determination about whether the report is valid or not. APS specialists in Texas are part of the state's protective agency called the Texas Department of Protective and Regulatory Services (TDPRS). Reports of abuse, neglect, and exploitation of the elderly and persons with disabilities are investigated by the TDPRS-APS. 
Reports are generally made by means of a 24 hour hotline; once a report is filed, an investigation must be initiated by the TDPRS-APS within 24 hours. Based on data from in-home investigations APS specialists are charged with determining whether a case is valid, invalid, or unable to be determined. Although their investigations are guided by standardized checklists, ultimately the finding of "neglect” is based on the APS specialist's judgment. In order to develop a rating scale that would capture these subjective judgments, we conducted structured interviews with 25 APS specialists in the greater Houston area to elicit the factors they considered in their determinations, such as events, client behaviors, and physical signs on which they based their findings of self-neglect.(Dyer, et al., in press). According to the responses of the 25 experienced APS specialists interviewed, elder neglect involves generally three discrete patient domains:

Upkeep of the environment: The inability to maintain the environment was the most frequently cited key element of self-neglect. This domain includes inability to clean the household and yard, as well as to manage the material goods acquired over the years.

Personal hygiene: The second most common element of neglect was a decline in personal hygiene. APS specialists often saw unkempt hair, clothing, nails and skin, lack of grooming, and indifference to usual social norms.

Cognition: Many specialists listed changes in cognition as an indicator of neglect, despite the fact that they do not routinely employ formal cognitive assessments in their evaluations. Impaired mental function seemed to sway the specialists when validating cases of neglect. Perhaps this reflects their adherence to the principle of client autonomy whereby neglect only exists, if one does not freely choose to live in less than desirable circumstances.

Instrument Development

Based on these findings, the research team developed an item pool that was distributed to an expert panel including three Adult Protective Service administrators, a civil attorney with expertise in elder mistreatment, an epidemiologist, a psychologist and a geriatrician. Panelists came from Texas, Washington DC, and Tennessee. These items are described in Table one. In addition to the three key areas identified above, the panel members cited nutritional status, 
failure to make use of medical care, failure to comply with medication use, and failure to maintain utilities as important components of self-neglect.

The research team struggled with the issue of assessing nutrition. The tools available to assess food intake generally rely on self-report obtained through interviews or food diaries. Accurate dietary intake data from free-living subjects is difficult to obtain. For obvious reasons, this process was deemed not to be feasible for elder self-neglectors. To attempt to document some aspect of food and nutrition, a home food storage survey was designed to assess five foods from each food group in the refrigerator, freezer, or pantry of the self-neglector. The assessment involved counting items in each storage area and randomly choosing items from each food group to evaluate expired or rotting food.

A final draft instrument was developed by Baylor research work group comprised of an epidemiologist, clinicians who frequently care for elders who are self-neglectors, and adult protective service workers. A rating scale of 0-4 was assigned for each of the assessment areas with specific descriptors to define the scale anchors. The workgroup determined that the tool should be employed in the home environment. The instrument was subsequently field tested and revised then piloted and revised once again.

\section{RESULTS}

\section{Field Testing}

A research assistant field tested the SSS for use in the home environment. She (J.L.) applied the test to ten self-neglectors, as defined by an APS assessment, and made extensive notes on the ease of administration of the tool. The field test showed that the test was easily administered in the home setting and took an average of 5 - 10 minutes to complete. Field testing also revealed that more clarity was needed regarding distinctions among the score levels ("0" 
through "4" for each item). Specifically, more detailed descriptors and/or pictures were essential to enable the user to rate the items effectively.

Enhanced descriptors of each item's object of assessment were developed and added. In addition, pictures were included adjacent to the " 0 " and " 4 " scores, located at the ends of the scale, for each item in the personal hygiene and environment domains. The CREST team also developed a detailed instruction manual for the SSS and trained a field research team consisting of a nurse practitioner and a research assistant for 2 hours each, then applied the SSS on ten additional patients, enabling the team to become facile in SSS administration. Members of the research team observed the CREST field team as they applied the tool in the home setting for three of the ten cases to insure that the tool was applied appropriately. Following this training period, a pilot study of the SSS in field application was undertaken.

\section{Pilot Study}

Subjects were elders reported for self-neglect whose cases were subsequently substantiated by Adult Protective Services (APS) and referred to the research team. Subjects were enrolled through a two step process whereby they initially signed a release allowing APS to refer them to Baylor researchers, then the CREST field team obtained informed consent at the homes of the subjects. Every subject had to demonstrate an understanding of the study by relaying back to the CREST field team members an understanding of the purpose of the study, their voluntary participation, and a summary of the procedures involved. After this pilot testing, the tool was further refined. The pilot testing revealed that there needed to be more items for environmental assessment, because in some cases, one or two rooms of the living quarters are in good repair or clean while the rest of the home was filthy or markedly cluttered. The CLOCK 
Test was added to determine the influence of cognition on functional outcomes or behaviors in the subjects.

The food storage survey did not work well. The survey did not take into account whether items were stored at appropriate temperatures or how to assess items with no expiration dates. In many instances, items from every food group were not available and items of food were found scattered throughout the house making it difficult to assess the number of items in specific food groups. As not all items had expiration dates, determination of the shelf-life of the food proved difficult, particularly as not all food groups were represented, therefore, randomly choosing items to assess expiration became complex and impractical.

\section{Current Tool}

The final tool is easy to administer and takes about 5 - 10 minutes; almost all of it can be completed through observation. The instrument has three sections: the first section (A) addresses personal appearance and assesses hygiene, the second section (B) is functional status which assesses instrumental activities of daily living that impact health and safety and the third section (C) involves an environmental assessment. Section A contains five items, Section B contains six items and Section C contains ten items. Each section is ranked from 0 which is normal to 4 which is severe self-neglect. The 0 and 4 rankings have an associated color picture where applicable to guide the administrator. The items for the revised tool are detailed in table two.

\section{DISCUSSION}

Overall, the pilot study findings suggest that the SSS is a promising measure for rapidly differentiating those persons who self-neglect from those who do not. Currently some APS agencies around the US use nonvalidated scales. Many agencies have no screening procedures and workers must rely on gut feelings. The concept of self-neglect is a complex one, consists of 
multiple domains, and assessment can be very subjective. For example, more experienced workers could become inured to findings that would alarm a less-experienced worker, leading to discrepancies in substantiating self-neglect. Additionally a less experienced worker may substantiate self-neglect in cases where it does not exist. The SSS was designed to provide an objective measure for identifying the presence and severity of self-neglect. Use of this tool could provide a common language to describe self-neglectors among the professionals who work with them in the same way that the Mini-Mental State Examination allows healthcare professionals to communicate effectively regarding cases of dementia.

The SSS is not designed to replace a careful history, physical examination or casework. Instead it is intended to serve as a screen for identifying at-risk persons and referring them for further intervention. It is also intended to convey the severity of the findings which constitute the prevalence of self-neglect. While in the end, the SSS did not include food intake/nutrition information, other studies of this population have documented nutritional deficiencies as a significant factor in self-neglectors (Smith et al., in press). The ability to differentiate among nutrient deficiencies may prove critical in treating these individuals appropriately (e.g., getting vitamin D to the vitamin D deficient, or vitamin B12 to the vitamin B12 deficient).

To date, development and use of the SSS have been productive. The ongoing studies include inter-rater reliability and validation of the tool against geriatric assessment. In the future, cross-validation of its performance, optimally using fresh samples of cases and controls drawn from the same populations as utilized in the pilot study, will further validate the SSS. In addition, studies that compare SSS performance across diverse populations will help inform development of improvements and modifications to the measure. If found to be widely applicable, the SSS could become a standard assessment tool for APS workers across the US and might be employed 
in clinics that routinely care for the elderly or demented persons who are clearly at risk. In addition, home health workers and hospice staff could employ this tool to detect and refer serious cases of self-neglect. In future studies, we hope to show that self-neglect is not only identifiable, but is also preventable and treatable. 
Table One: Initial Item Pool for the SSS

\section{Personal hygiene}

Hair -cleanliness and grooming

Skin - including nails

Odors - fecal or urinary incontinence, gangrenous flesh

Infestations - lice, scabies, maggots

Clothing - cleanliness

\section{Environment}

Bath

Kitchen

Main living areas

Pets

Presence of urine or feces

\section{Functional ability}

Shopping

Cleaning clothes

Handling Finances

Accessing Healthcare

Obtaining meals

Clean

Using the telephone $\underline{\text { Table Two: Current Item Pool for the SSS }}$

SECTION A: Personal hygiene assessment

Hair

Nails

Skin

Insect infestations

Clothing

SECTION B: Assessment of cognitive, health and safety issues

Cognitive test - Clock exam

Evidence of delusional state

Response to emergency

Evidence of usual source of medical care

Time since last visit to physician

Evidence of untreated health conditions

\section{Section C: Environmental assessment}

Exterior condition

$\begin{array}{ll}\text { Yard/sidewalk } & \text { Roof } \\ \text { Windows } & \text { Walls }\end{array}$

Interior condition:

Odor Clutter

Cleanliness

Structure

Pets

Utilities 


\section{References}

Dyer, C.B., Pavlik, V.N., Murphy, K.P., \& Hyman, D.J. (2000). The high prevalence of depression and dementia in elder neglect. Journal of the American Geriatrics Society. 48 (2), 2025-208.

Dyer, C.B., Connolly, M.T., \& McFeeley, P. (2002). The clinical and medical forensics of elder abuse and neglect. In R. J. Bonnie \& R.B.Wallace (Eds.), Elder Mistreatment: Abuse, neglect and exploitation in an aging America. (pp. 339-381). Washington DC: National Academic Press.

Dyer, C.B., Pavlik, V.N., Toronjo, C., Cunningham, M., Poythress, E.L., Searle, N., et al. (in press). The Key elements of self-neglect. Journal of Elder Abuse and Neglect.

Fulmer, T., Dyer, C.B., Connolly, M.T., Guadango, L. (2004). Progress in elder abuse assessment instruments. Journal of the American Geriatrics Society. 52 (2), 297-304.

Lachs, M.A., Williams, C.S., O’Brien, S., Pillemer, K.A., Charlson, M.E. (1998). Mortality of elder mistreatment. Journal of the American Medical Association, 280(5), 428-432.

Pavlik, V.N., Festa, N.A., Hyman, D.J., \& Dyer, C.B. (2001). Quantifying the problem of abuse and neglect in adults-analysis of a statewide database. Journal of the American Geriatrics Society,49, 45-48.

Smith, S.M., Mathews, Oliver S.A., Zwart, S.R., Kala, G., Kelly, P.A., et al. (in press). Nutritional status in self-neglecting elderly Journal of Nutrition.

Teaster, P.B., Dugar, T.A., Mendiondo, M.S., Abner, E.L., Cecil, K. (2005). The 2004 Survey of state adult protective services: Abuse of adults 60 years of age and older. The National Center on Elder Abuse. Retrieved on March 18, 2006 from http://www.elderabusecenter.org/pdf/2-14-06\%20FINAL\%2060+REPORT.pdf 